\title{
EXPERIMENTOS DIDÁTICOS ENVOLVENDO RADIAÇÃO MICROONDAS
}

\author{
Fabiana Rosini, Clésia C. Nascentes* e Joaquim A. Nóbrega \\ Departamento de Química, Universidade Federal de São Carlos, CP 676, 13560-970 São Carlos - SP
}

Recebido em 23/7/03; aceito em 15/4/04; publicado na web em 27/8/04

\begin{abstract}
MICROWAVE-ASSISTED EXPERIMENTS FOR UNDERGRADUATE COURSES. Theoretical and practical aspects of the use of microwave-assisted strategies in chemistry are introduced for students using simple and safe experiments employing a domestic oven. Three procedures are proposed for evaluating the distribution of microwave radiation inside the microwave oven cavity: (1) variation of the volume of marshmallows; (2) drying of filter paper wetted with Co(II) solution, and (3) variation of water temperature, after microwave-assisted heating. These experiments establish the position with the highest incidence of microwave radiation in the oven cavity, which was chosen for the synthesis of salicylic acid acetate. This synthesis was performed in $5 \mathrm{~min}$ of heating and the yield was around $85 \%$. All experiments can be carried out in a $4 \mathrm{~h}$ lab-session using low-cost instrumentation.
\end{abstract}

Keywords: microwave radiation distribution; microwave-assisted synthesis; salicylic acid acetate.

\section{INTRODUÇÃO}

O desenvolvimento da tecnologia de microondas ocorreu principalmente durante a II Guerra Mundial. Os fornos de microondas começaram a ser utilizados para aquecimento de alimentos na década de 50, e a partir dos anos 80, esse tipo de energia passou a ser utilizado em laboratórios de química ${ }^{1}$.

Em química, os fornos de microondas são utilizados para diversas finalidades, tais como secagem de amostras ${ }^{2,3}$, sínteses ${ }^{4,5}$, extração de compostos orgânicos ${ }^{6,7}$ e mineralização de amostras orgâni$\operatorname{cas}^{8-10}$ e inorgânicas ${ }^{11,12}$.

Com essa diversidade de aplicações torna-se relevante discutir esse tópico em aulas teóricas e práticas de química. Os princípios envolvidos no aquecimento por microondas englobam conceitos químicos e físicos, tais como temperatura, ligação química, estrutura molecular, momento de dipolo, polarização, capacidade calorífica e constante dielétrica ${ }^{13}$. O forno de microondas é um equipamento conhecido pela maioria dos estudantes, mas os conceitos químicos envolvidos em seu funcionamento e o grande número de aplicações que esses equipamentos têm em química não são tão disseminados.

Este trabalho propõe experimentos utilizando um forno de microondas doméstico para aplicações em laboratório. Aspectos da teoria sobre microondas são sucintamente apresentados visando melhor compreensão do funcionamento dos fornos de microondas. A aula prática é dividida em duas partes: primeiramente executa-se o mapeamento térmico do forno por diferentes procedimentos e, após o mapeamento, a posição na qual ocorre a maior incidência de radiação microondas na cavidade do forno é utilizada para a síntese de aspirina, i.e., ácido acetilsalicílico.

\section{Teoria de microondas}

A radiação microondas é um tipo de energia eletromagnética com freqüência na faixa de $10^{3}$ a $10^{4} \mathrm{MHz}$. Essa radiação nãoionizante causa migração de íons e rotação de dipolos, mas não causa mudanças na estrutura molecular ${ }^{1}$.
O aquecimento de um material por irradiação com microondas se dá devido à interação da onda eletromagnética com o dipolo elétrico da molécula. Um importante atributo do aquecimento por microondas é a absorção direta da energia pelo material a ser aqueci$\mathrm{do}^{14}$, ao contrário do que ocorre quando o aquecimento é realizado por convecção, no qual a energia é transferida lentamente do recipiente de reação para a solução. Assim, o aquecimento por microondas é seletivo e dependerá, principalmente, da constante dielétrica e da freqüência de relaxação do material ${ }^{15}$.

Para entender simplificadamente o aquecimento de uma substância no forno de microondas, pode-se fazer uma analogia ao que acontece quando as moléculas são submetidas à ação de um campo elétrico. Quando esse é aplicado, as moléculas que possuem momento de dipolo elétrico ou que podem ter momentos dipolares induzidos tendem a se alinhar com o campo e quando o campo elétrico é removido, ocorrerá uma relaxação dielétrica, ou seja, as moléculas tenderão a retornar ao seu estado não alinhado, dissipando a energia absorvida na forma de calor ${ }^{1,15}$. A princípio, quanto maior for o dipolo elétrico, mais intensa deve ser a orientação molecular sob a ação do campo elétrico. Se um material possui um valor maior de constante dielétrica, a princípio, maior quantidade de energia pode ser armazenada. Em um campo de fases alternadas, como é o caso de uma onda eletromagnética, a orientação molecular varia ciclicamente, sendo que para um forno de microondas com frequiência de $2450 \mathrm{MHz}$, que é a frequiência adotada em fornos domésticos e laboratoriais, ocorrem $10^{9}$ orientações $/ \mathrm{s}^{13}$.

Um forno de microondas típico para uso em laboratório possui seis componentes principais: o gerador de microondas (chamado magnetron), o guia de ondas, a cavidade do forno, o espalhador de ondas, um sistema de ventilação e um rotor $^{1}$. Os fornos de microondas domésticos não possuem o sistema de ventilação e o rotor.

Devido ao alto custo dos equipamentos desenvolvidos para aplicações laboratoriais, freqüentemente são empregados fornos de microondas domésticos. Esses equipamentos não possuem uma distribuição uniforme da radiação microondas, pois não foram projetados para tal finalidade. Eles produzem interferência entre as microondas e, com isso, algumas partes do forno recebem maior incidência de ondas que outras ${ }^{15}$. Assim, é necessário mapear a distribuição da radiação microondas para uma utilização mais efetiva da energia gerada. Além disso, os fornos de microondas desenvolvidos para traba- 
lhos laboratoriais possuem dispositivos de segurança como, por exemplo, sensores de gases na cavidade, porta retrátil para alívio de pressão e auto-fechamento e frascos reacionais capazes de suportar elevadas pressões (até $100 \mathrm{~atm}$ ), altas temperaturas (até $260^{\circ} \mathrm{C}$ ) e inércia química frente a ácidos concentrados. Esses dispositivos são essenciais para implementação de procedimentos agressivos de digestão de amostras. Entretanto, experimentos simples e demonstrações didáticas podem ser conduzidos com segurança em fornos de microondas projetados para uso doméstico.

\section{PARTE EXPERIMENTAL}

\section{Mapeamento do forno de microondas}

O forno de microondas utilizado em todos os experimentos é da marca Brastemp, $220 \mathrm{~V}, 28 \mathrm{~L}$, potência máxima de $700 \mathrm{~W}$ e opera sob uma freqüência de $2450 \mathrm{MHz}$.

\section{Mapeamento com "marshmallows"}

Distribuiu-se os "marshmallows" de forma simétrica dentro da cavidade do forno e irradiou-se à potência máxima por $40 \mathrm{~s}$. Observou-se o aumento de volume dos mesmos. Esse experimento foi realizado com e sem o prato giratório. A escolha de "marshmallows" para esse experimento pode ser justificada pela variação de volume que ocorre nesse alimento poroso, quando ligeiramente aquecido. Entretanto, deve-se evitar aquecimento excessivo, que causaria caramelização devido ao alto teor de açúcar.

\section{Mapeamento por meio da variação da temperatura da água}

Para este experimento foram utilizados béqueres de vidro de 250 $\mathrm{mL}$ e um termômetro com variação de temperatura de $0-200{ }^{\circ} \mathrm{C}$.

Colocou-se $200 \mathrm{~mL}$ de água nos béqueres e mediu-se a temperatura antes da irradiação. Cinco béqueres foram dispostos simultaneamente dentro da cavidade do forno, da forma mais simétrica possível, e irradiados por $120 \mathrm{~s}$ à potência máxima. Após o aquecimento, a temperatura final da água foi rapidamente medida em cada posição.

Procedimento similar foi adotado para o estudo das posições separadamente. Para isso foram fixadas nove posições dentro do forno e aqueceu-se apenas 1 béquer por vez.

A disposição dos béqueres em ambos os experimentos está apresentada na Figura 1.

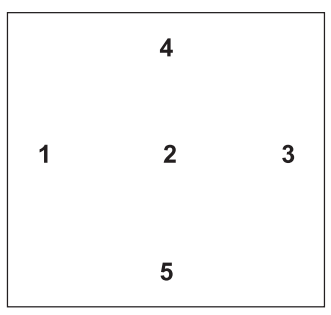

(a)

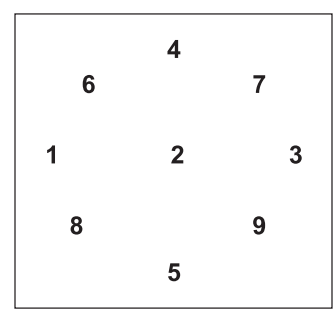

(b)
Figura 1. Disposição dos béqueres na cavidade do forno de microondas no mapeamento: (a) cinco posições simultâneas e (b) nove posições separadamente

Mapeamento por meio da secagem da solução aquosa de $\mathrm{CoCl}_{2}$

O material utilizado neste experimento é um papel absorvente utilizado para cobrir bancadas, e o reagente $\mathrm{CoCl}_{2}$. $6 \mathrm{H}_{2} \mathrm{O}$ (Synth, Diadema, SP).
Preparou-se uma solução de cloreto de cobalto(II) $4 \% \mathrm{~m} / \mathrm{v}$, dissolvendo-se $4 \mathrm{~g}$ do sal em água desionizada. Depois transferiu-se quantitativamente essa solução para um balão volumétrico de $100 \mathrm{~mL}$ e completou-se o volume com água desionizada.

Recortou-se o papel absorvente com o mesmo diâmetro do prato giratório. Esse papel foi fixado no prato e embebido com a solução de $\mathrm{CoCl}_{2} 4 \% \mathrm{~m} / \mathrm{v}$. Colocou-se o prato giratório com o papel embebido no forno e irradiou-se à potência máxima por $3 \mathrm{~min}$. Retirou-se o prato e observaram-se os pontos de maior irradiação. $\mathrm{O}$ experimento foi realizado com o prato girando e com o prato fixo na cavidade.

\section{Aplicação: síntese de aspirina}

A síntese da aspirina foi realizada de acordo com procedimento utilizado nas práticas de química geral ${ }^{16}$, sendo que a modificação consiste em substituir o aquecimento convencional em banho maria por aquecimento assistido por microondas.

Pesou-se aproximadamente $5 \mathrm{~g}$ de ácido salicílico e colocou-se em um béquer de $250 \mathrm{~mL}$. Adicionou-se $5 \mathrm{~mL}$ de anidrido acético, distribuído uniformemente por todo o sólido. $\mathrm{O}$ béquer contendo a mistura foi coberto com um vidro de relógio e colocado na posição otimizada do prato giratório (Figura $1 \mathrm{~b}$, posição 2, na qual o mapeamento indicou maior incidência de radiação microondas). No canto direito do forno (ponto de menor irradiação) colocou-se um outro béquer com 200 mL de água para absorção da radiação microondas excedente. Irradiou-se à máxima potência durante aproximadamente $5 \mathrm{~min}$, até que a mistura reacional atingisse $120^{\circ} \mathrm{C}$, sendo a temperatura determinada com um termômetro. $\mathrm{O}$ béquer foi cuidadosamente retirado do forno. Deixou-se que o mesmo resfriasse até 85-90 ${ }^{\circ} \mathrm{C}$. A mistura oleosa foi transferida para um outro béquer contendo água fria para recristalização. A mistura foi mantida em banho de gelo até a formação dos cristais e depois foi filtrada à vácuo, sendo o sólido lavado com água fria. O sólido foi seco completamente em estufa de circulação forçada e sua massa determinada. Para comprovação da pureza foi realizado um teste para salicilato com solução de $\mathrm{FeCl}_{3}{ }^{17} \mathrm{e}$ o ponto de fusão foi determinado. Como o teste para salicilato foi positivo, procedeu-se à recristalização do produto sólido. Para isso, adicionou-se um volume de $7 \mathrm{~mL}$ de 2-propanol ao sólido obtido e aqueceu-se a mistura até a total dissolução dos cristais. Retirou-se a mistura do aquecimento e a mesma foi resfriada até a recristalização.

\section{RESULTADOS E DISCUSSÃO}

\section{Mapeamento do forno de microondas}

Os experimentos envolvendo o mapeamento da distribuição da radiação na cavidade do forno de microondas visaram determinar o ponto de maior incidência da irradiação e utilizar esse dado para a síntese de aspirina. Em todos os experimentos os valores adotados para tempo de aquecimento e potência aplicada refletem as características do material que irá absorver a radiação microondas e a aplicação visada.

\section{Mapeamento com "marshmallows"}

"Marshmallows" foram distribuídos uniformemente na cavidade do forno e irradiados. Esse experimento foi proposto por Whittaker ${ }^{14}$, como uma forma qualitativa, simples e rápida de verificar que a distribuição da radiação microondas não é uniforme na cavidade do forno. Isso ocorre porque em um forno de microondas, uma pequena parte das ondas é absorvida pelas paredes metálicas e a maior parte é refletida pelas paredes do forno, podendo ocorrer in- 
terferência. Em alguns pontos ocorrem interferências construtivas para gerar ondas de alta intensidade (anti-ânodos) e em outros pontos ocorrem interferências destrutivas ${ }^{14}$.

Na Figura 2 pode-se observar que depois de irradiados, alguns "marshmallows" triplicaram de volume e quando o prato giratório não foi utilizado (Figura 2d) o aquecimento excessivo causou a caramelização de alguns "marshmallows" nos pontos de maior incidência de radiação microondas. Por outro lado, outros "marshmallows" permaneceram quase inalterados, indicando uma baixa incidência de radiação nesses pontos. $\mathrm{O}$ aumento de volume foi mais uniforme quando se utilizou o prato giratório, que proporcionou uma distribuição de radiação mais homogênea na cavidade do forno. Esse aumento de volume ocorre, pois os "marshmallows" são porosos e com o aquecimento ocorre uma expansão do ar que ocupa os poros, com a conseqüente expansão dos "marshmallows".

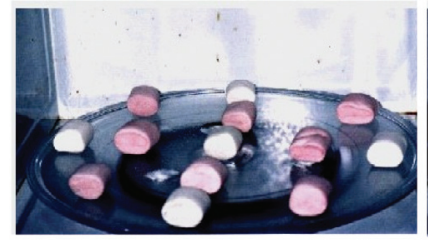

a

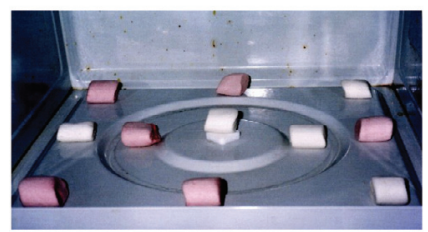

C

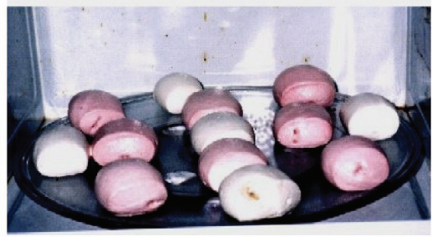

b

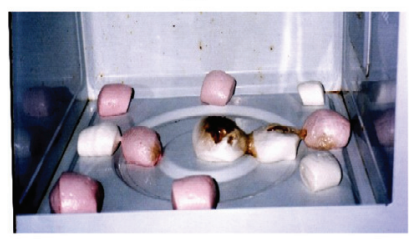

d
Figura 2. Fotos obtidas no mapeamento realizado com "marshmallows": (a) antes da irradiação das microondas com prato giratório; (b) após irradiação com prato giratório; (c) antes da irradiação das microondas, sem prato giratório e (d) após a irradiação sem prato giratório

Esse comportamento observado para a distribuição de radiação reflete o comportamento esperado e o experimento com "marshmallows" possibilita a obtenção de uma rápida imagem qualitativa sobre a distribuição de radiação microondas no interior da cavidade.

\section{Mapeamento por meio da variação da temperatura da água}

O aumento da temperatura da água é diretamente proporcional à incidência de radiação microondas. Por meio da variação da temperatura, pode-se calcular a potência do forno de microondas em $\mathrm{J} \mathrm{s}^{-1}$ que corresponde a Watts no sistema internacional de unidades. A massa de água utilizada nos experimentos ( $200 \mathrm{~g}$ ) corresponde a 11,11 mols.

$$
\mathrm{P}=\frac{\mathrm{Cp} \mathrm{n} \Delta \mathrm{T}}{\mathrm{t}}
$$

sendo: $\mathrm{Cp}=$ capacidade calorífica da água $\left(75,312 \mathrm{~J} \mathrm{~K}^{-1} \mathrm{~mol}^{-1}\right) ; \mathrm{n}=$ quantidade de matéria de água utilizada (mols); $\Delta \mathrm{T}=$ temperatura final - temperatura inicial e $\mathrm{t}=$ tempo de aquecimento $(\mathrm{s})$

Os resultados obtidos neste mapeamento estão apresentados na Figura 3. Quando os béqueres foram irradiados simultaneamente, observa-se menor variação de temperatura em todos os pontos, pois ocorre uma distribuição da energia irradiada entre os béqueres (Figura 3a). Neste experimento, a posição com maior incidência de radiação microondas foi a 3 (Figura 1). A potência calculada variou de 70,7 W (posição 5) até 195,2 W (posição 3), indicando uma vari- (a)

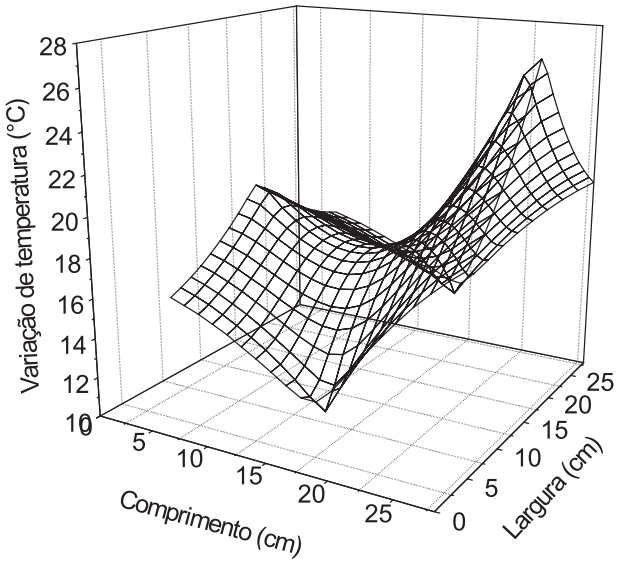

(b)

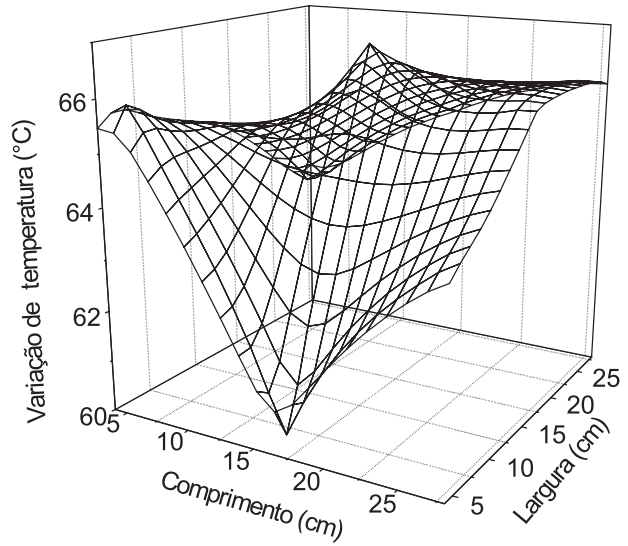

Figura 3. Superfícies de resposta obtidas no mapeamento do forno de microondas por meio da variação de temperatura da água: (a) 5 béqueres simultaneamente e (b) uma posição por vez.

ação de $64 \%$. Na Figura 3b pode-se observar que ao irradiar os béqueres separadamente, a variação de temperatura foi maior e o ponto de máxima incidência de radiação microondas foi diferente do primeiro experimento, sendo a posição 2 (central) o ponto de maior incidência. Isso pode ser devido às reflexões das microondas, que podem ocorrer nas paredes do forno. As potências calculadas variaram de 418,4 W (posição 5) até 467,2 W (posição 2), indicando uma variação de $10,5 \%$.

\section{Mapeamento por meio da secagem de solução aquosa de $\mathrm{CoCl}_{2}$}

Os resultados obtidos neste mapeamento podem ser observados na Figura 4. O cloreto de cobalto(II) quando hidratado possui coloração rosa (Figura 4a) e quando desidratado torna-se azul ${ }^{17}$. Essa

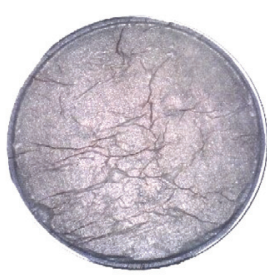

a

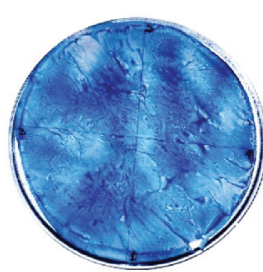

b

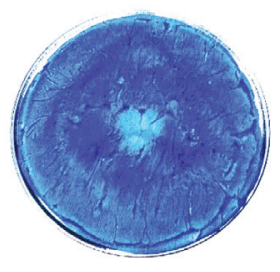

C
Figura 4. Fotos obtidas no mapeamento realizado por meio da secagem da solução aquosa de $\mathrm{CoCl}_{2}$ : (a) antes da irradiação das microondas; (b) após irradiação com prato fixo e (c) após irradiação com prato giratório 
propriedade dos sais de Co(II) é bem conhecida e justifica a escolha desse cátion para o experimento proposto. A variação de coloração possibilita um rápido mapeamento qualitativo do processo de secagem assistido por radiação microondas e inferir a distribuição de radiação microondas na cavidade.

Nos pontos nos quais a incidência de radiação foi maior, pode-se observar uma coloração azul mais intensa (Figuras $4 b$ e 4c). Diferenças também foram observadas quando o experimento foi realizado com o prato girando e com o prato fixo, sendo que com o prato girando (Figura 4c) a coloração azul intensa é mais uniforme ao redor do centro do prato, pois a rotação do mesmo colabora para melhor homogeneidade na distribuição da radiação microondas. Quando o prato foi mantido fixo, percebe-se uma coloração azul mais pontual (Figura 4b).

Apesar dos procedimentos propostos para o mapeamento serem diferentes, de forma geral, indicam a mesma região do forno como sendo a de maior incidência da radiação microondas. A comparação é mais facilmente realizada entre o procedimento que envolve a variação de volume dos "marshmallows" e o da variação da temperatura da água, nos quais o estudo foi realizado em pontos determinados na cavidade. Verifica-se que, em ambos, na região central e à direita do centro ocorreu a maior incidência de microondas.

A vantagem do procedimento com "marshmallows" é a possibilidade de um mapeamento qualitativo rápido e visual da distribuição de radiação. Por outro lado, o procedimento utilizando água possibilita uma quantificação da heterogeneidade de distribuição da radiação pela medida de variação de temperatura em cada posição. Tentou-se medir a variação de volume dos "marshmallows" após o aquecimento, porém essa estratégia não foi viável pois além de ter que trabalhar com um material aquecido, também ocorria uma brusca contração dos "marshmallows" durante o processo de resfriamento.

No mapeamento utilizando a solução aquosa de $\mathrm{CoCl}_{2}$, toda a superfície sobre o prato giratório foi avaliada e isso implicou que os resultados obtidos diferem dos obtidos pelos outros procedimentos. De acordo com esse procedimento, a incidência de microondas é baixa na região central da cavidade (Figura 4c).

\section{Aplicação: síntese de aspirina}

O ácido acetilsalicílico (AAS), comercialmente conhecido como aspirina, pode ser sintetizado pela reação do ácido salicílico com o anidrido acético sob aquecimento. Os produtos dessa reação são o ácido acetilsalicílico e o ácido acético.

Este experimento propõe uma nova forma de aquecimento para a reação, o aquecimento assistido por radiação microondas.

Os rendimentos obtidos para a síntese de aspirina utilizando aquecimento por microondas e aquecimento convencional são apresentados na Tabela 1. Pode-se observar que o procedimento proposto foi mais eficiente que o tradicional, que utiliza aquecimento em banho-

Tabela 1. Resultados obtidos na síntese da aspirina utilizando aquecimento por radiação microondas e aquecimento convencional

\begin{tabular}{lccc}
\hline Experimento & $\begin{array}{c}\text { Ponto } \\
\text { Fusão }\left({ }^{\circ} \mathrm{C}\right)\end{array}$ & $\begin{array}{c}\text { Massa } \\
(\mathrm{g})\end{array}$ & $\begin{array}{c}\text { Rendimento } \\
(\%)\end{array}$ \\
\hline $\begin{array}{l}\text { Radiação } \\
\text { microondas }\end{array}$ & $133-134$ & 5,579 & 85 \\
$\begin{array}{l}\text { Aquecimento } \\
\text { convencional }{ }^{17}\end{array}$ & $133-134$ & 4,236 & 65 \\
\hline
\end{tabular}

* O ponto de fusão esperado é de $132-134{ }^{\circ} \mathrm{C}^{18}$. maria e é normalmente realizado nas disciplinas experimentais dos cursos de graduação. O tempo de aquecimento no procedimento convencional é de 30-40 min e no procedimento proposto, $5 \mathrm{~min}$. Como discutido anteriormente, a energia microondas é absorvida diretamente pelo meio reacional, e isso proporciona um aquecimento mais rápido e uniforme ${ }^{14}$. $\mathrm{O}$ aquecimento assistido por microondas reduziu o tempo consumido na prática e proporcionou um maior rendimento do produto final. $\mathrm{O}$ ponto de fusão para o produto obtido pelos dois procedimentos de aquecimento avaliados é próximo ao valor estabelecido na literatura ${ }^{18}$ para o ácido acetilsalicílico.

\section{CONCLUSÕES}

Discussões sobre a utilização da energia de microondas em cursos de graduação são importantes, pois essa radiação vem sendo cada vez mais aplicada em diferentes campos da química.

Todos os experimentos aqui propostos para o mapeamento de fornos de microondas são simples, apresentam baixo custo e são relativamente rápidos, podendo ser facilmente implementados em aulas práticas. Sua execução, além de indicar como o forno de microondas deve ser utilizado para aplicações futuras, ajuda o aluno a compreender melhor o funcionamento dos fornos de microondas.

A síntese da aspirina é apenas um exemplo de como a energia de microondas pode ser aplicada em práticas de química, tornando-as mais simples e rápidas.

Todos esses experimentos podem ser conduzidos com segurança em uma aula experimental de $4 \mathrm{~h}$ e envolvem equipamentos de baixo custo.

\section{AGRADECIMENTOS} dos.

Ao CNPq, CAPES e FAPESP pelos auxílios e bolsas concedi-

\section{REFERÊNCIAS}

1. Kingston, H. M.; Jassie, L. B., eds.; Introduction to Microwave Sample Preparation, ACS: Washington, 1988.

2. Reh, C. T.; Gerber, A.; Food Chem. 2003, 82, 125.

3. Ortiz, A. I. C.; Albarran, Y. M.; Rica, C. C.; J. Anal. At. Spectrom. 2002, 17,1595 .

4. Rao, V. V. V. N. S. R; Ravikanth, S.; Reddy, G. V.; Maitraie, D.; Yadla, R.; Rao, P. S.; Synth. Commun. 2003, 33, 1523.

5. Subassi, R.; Mathews, T.; Swaminathan, K.; Sreedharan, O. M.; J. Alloys Compd. 2003, 354, 193.

6. Concha-Grana, E.; Barriada-Pereira, M.; Turnes-Carou, M. I.; MuniateguiLorenzo, S.; Lopez-Mahia, P.; Rodriguez, D. P.; Anal. Bioanal. Chem. 2003, 375,1225

7. Shu, Y. Y.; Ko, M. Y.; Chang, Y. S.; Microchem. J. 2003, 74, 131

8. McCarlthy, H. T.; Ellis, P. C.; J. Assoc. Off. Anal. Chem. 1991, 74, 566.

9. Alonso, E. V.; de Torres, A. G.; Pavon, J. M. C.; Analyst 1992, 117, 1157.

10. Costa, L. M.; Silva, F. V.; Gouveia, S. T.; Nogueira, A. R. A.; Nóbrega, J. A.; Spectrochim. Acta, Part B 2001, 56, 1981.

11. Klock, P. R.; Lamothe, P. J.; Talanta 1986, 33, 495.

12. Rantala, R. T. T.; Loring, D. H.; Anal. Chim. Acta 1989, 220, 263.

13. Watkins, K. W.; J. Chem. Educ. 1983, 60, 1043.

14. Whittaker, A. G., Educ. Chem. 2002, Sep., 134.

15. Barboza, A. C. R. N.; Cruz, C. V. M. S.; Graziani, M. B.; Lorenzetti, M. C. F.; Sabadini, E.; Quim. Nova 2001, 24, 901.

16. Silva, R. R.; Bocchi, N.; Rocha Filho, R. C.; Introdução à Química Experimental, McGraw Hill: São Paulo, 1990.

17. Vogel, A.; Química Analítica Qualitativa, Trad. de Antonio Gimeno, Mestre Jou: São Paulo, 1981.

18. Weast, R. C., ed.; Handbook of Chemistry and Physics, $64^{\mathrm{a}}$ ed.; CRC Press: Boca Raton, 1984. 